\title{
Nuclear Physics from Lattice QCD: The Spectrum, Structure and Interactions of Hadrons
}

\author{
Colin Morningstar ${ }^{1}$, John W. Negele ${ }^{2}$, Kostas $\operatorname{Orginos}^{3,4}$, David Richards ${ }^{4}$, \\ and Martin J. Savage ${ }^{5}$ \\ ${ }^{1}$ Department of Physics, Carnegie-Mellon University, Pittsburgh, PA 15213 \\ ${ }^{2}$ Center for Theoretical Physics, Massachusetts Institute of Technology, \\ Cambridge, MA 02139 \\ ${ }^{3}$ Department of Physics, College of William and Mary, Williamsburg, \\ VA 23187 \\ ${ }^{4}$ Thomas Jefferson National Accelerator Facility, Newport News, VA 23606 \\ ${ }^{5}$ Department of Physics, University of Washington, Seattle, WA 98195
}

\begin{abstract}
The importance of lattice QCD to our understanding of the structure, spectroscopy, and interaction of hadrons is decribed. Recent accomplishments in each of these areas is outlined, and the opportunities emerging with increasing computational power are identified. Milestones at the 10 Tflops-years, 100 Tflops-years and Petaflops-years scales are presented.
\end{abstract}

\section{Motivation}

Understanding how the structure, spectroscopy, and interactions of hadrons emerge from QCD is one of the central challenges of contemporary nuclear physics. Recent advances in lattice field theory, developments in computer technology and investment in computer resources for fundamental QCD research have now made lattice QCD a powerful quantitative tool that provides an unprecedented opportunity to understand the phenomena arising from QCD from first principles and to make precision calculations of the predictions of QCD.

The internal quark and gluon structure of the nucleon is a defining component of hadronic physics just as the structure of the hydrogen atom is of atomic physics. Hence, one goal of lattice QCD is precision calculation of fundamental experimental quantities characterizing the nucleon. These include form factors specifying the distribution of charge and current 
and how constituents interact to recoil together at high momentum transfer, moments of parton densities, helicity, and transversity distributions as a function of momentum fraction, and the moments of generalized parton distributions (GPD's). The calculations of all of these are specified as Nuclear Physics 2014 milestones in Hadronic Physics (HP). These first principles calculations will have direct impact on key experimental measurements at JLab and RHIC-spin, including 1) the HP 2008 milestones of measuring the spin carried by glue in the proton and extracting accurate information on generalized parton distributions, 2) the HP 2010 milestones of determining four EM form factors to $Q^{2}=3.5 \mathrm{GeV}^{2}$ and electroweak form factors to $1 \mathrm{GeV}^{2}$, and 3) the HP 2013 milestone of measuring flavor-identified $q$ and $\bar{q}$ contributions to the proton spin.

Another essential goal in hadron structure is obtaining insight into how QCD works. How does the spin of the nucleon arise from the helicity and orbital angular momentum of quarks and of gluons? What is the fundamental mechanism for confinement and what is the role of diquarks and instantons in hadrons? How does hadron structure change as one varies parameters that cannot be varied experimentally, such as the number of colors, the number of flavors, the quark mass, or the gauge group? Finally, once lattice calculations are validated by extensive comparison with directly comparable experiments, one can exploit lattice QCD to provide information complementary to experiment. For example, the moments of GPD's calculated on the lattice and the convolutions of GPD's measured experimentally can be combined to constrain GPD's far more effectively than either experiment or lattice QCD could separately.

A detailed knowledge of the meson and baryon spectra from first principles will distill the key degrees of freedom needed to describe the bound states of the theory. The complete combined analysis of available experimental data on the photoproduction of nucleon resonances is the Nuclear Physics 2009 milestone in Hadronic Physics (HP), and the measurement of the electromagnetic properties of the low-lying baryons is an HP 2012 milestone. The so-called hybrid mesons, a new form of excited state in which excitations of the gluon field play an explicit structural role, are especially interesting, and the GlueX Collaboration proposal to seek information about exotic mesons is a flagship component of the $12 \mathrm{GeV}$ upgrade at Jefferson Laboratory.

The current intense experimental efforts in hadron spectroscopy demand a commensurate effort to predict and understand the hadron spectrum from first-principles calculations using lattice QCD. This interplay between theory an experiment is essential to the interpretation of resonance data, and to extract the greatest benefit from the Department of Energies facilities.

A grand challenge for strong interaction physics is to be able to rigorously compute the properties and interactions of nuclei. The many decades of theoretical and experimental investigations in nuclear physics have, in many instances, provided a very precise phenomenology of the strong interactions in the non-perturbative regime. However, at this point we have little understanding of much of this phenomenology in terms of QCD. Any nuclear observable is essentially a function of only five constants, the length scale of the strong interactions, $\Lambda_{\mathrm{QCD}}$, the quark masses, $m_{u}, m_{d}, m_{s}$, and the electromagnetic coupling, $\alpha_{e}$; at low energies the dependence upon the top, bottom and charm quarks masses is encapsulated in $\Lambda_{\mathrm{QCD}}$.

The way to proceed toward nuclei is to establish a small number of effective theories, each 
of which has well-defined expansion parameters and can be shown to be the most general form consistent with the symmetries of QCD. Each theory must provide a complete description of nuclei over some range of atomic number. Calculations in two "adjacent" theories are performed for a range of atomic numbers for which both theories converge. One then matches coefficients in one EFT to the calculations in the other EFT or to the lattice, and thereby one can make an indirect, but rigorous connection between QCD and nuclei. Although significant development needs to be done, it appears that several different calculational regimes emerge: Lattice QCD, Exact Many-Body Methods, Coupled Cluster Calculations, and Density Functional Theory, and Very Large Nuclei. Once this calculational scheme has been put into place, or even just those components adjacent to lattice QCD, reliable calculations of strong interaction processes in regimes where experiment is not feasible become possible. A great example of this is the interaction between hyperons and nucleons, which plays an important role in determining the nuclear equation of state in the interior of a neutron star.

\section{Accomplishments}

Accomplishments over the last five years have established the methodology and laid the groundwork for hadron structure, spectroscopy, and hadron scattering calculations. Because of the cost of full QCD calculations with light quarks in the chiral regime, initial calculations were restricted to the "heavy pion" domain of pion masses in excess of $500 \mathrm{MeV}$. In this domain, form factors, the lowest three moments of quark, spin, and transversity distributions[1], and generalized form factors corresponding to the lowest three moments of generalized parton distributions have been calculated $[2,3]$. Salient achievements include separating the contributions of the quark spin and orbital angular momentum to the nucleon spin [2] and observing strong dependence of the transverse size of the nucleon on the longitudinal momentum fraction [3]. The transition form factors between the nucleon and Delta has been calculated to explore the role of deformation $[4,5]$.

In spectroscopy, techniques to calculate extended sources within the appropriate representation of the hypercubic group have been developed and utilized to calculate ground and excited states in each symmetry channel [6, 7], and evidence for pentaquark states sought [8, 9]. Finally, the realization was made that lattice QCD computations in foreseeable future could be used to derive rigorous results for nuclear physics[10], thus opening an important new avenue for lattice computations that are at the core of this whitepaper.

An essential step toward the chiral regime with light quarks has recently been taken using a hybrid calculation combining computationally economical staggered sea quark configurations generated by the MILC collaboration and domain wall valence quarks that have lattice chiral symmetry. The axial charge[12], moments of structure functions and generalized form factors[13], and the axial $\mathrm{N}$ to Delta transition form factors[14] have been calculated for pion masses as light as $350 \mathrm{MeV}$. Hybrid calculations of the pion form factor were also performed [15]. A number of meson-meson scattering lengths, $I=2 \pi \pi$ [11], $K \pi$ [16] and $K K$, have been determined with reasonable precision at pion masses down to $m_{\pi} \sim 290 \mathrm{MeV}$. The first calculations of nucleon-nucleon scattering [17] and hyperon-nucleon scattering [18] 


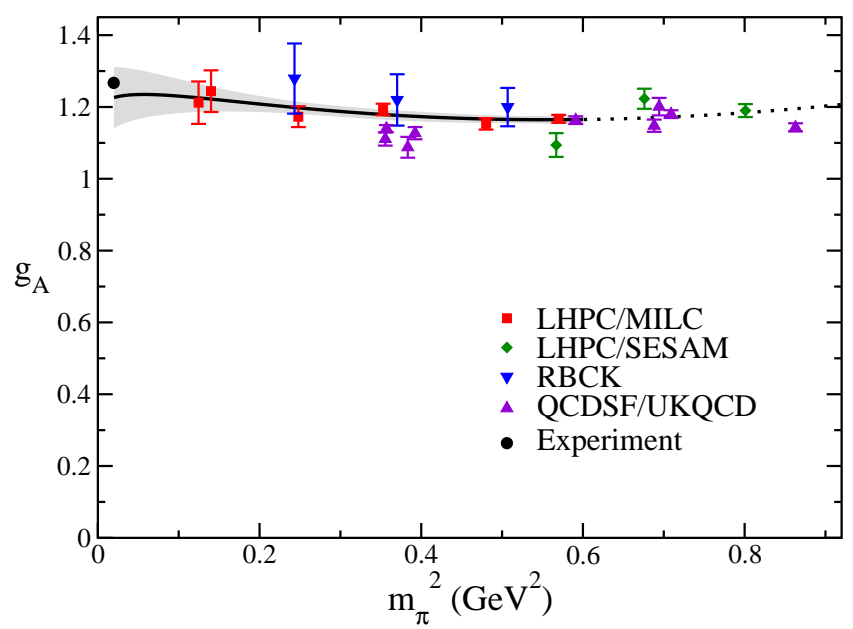

Figure 1: Chiral extrapolation of full QCD calculations of $g_{A}$. The solid line and error band denote the infinite volume $\chi \mathrm{PT}$ fit to the LHPC/MILC results shown in red. Note that all the other full QCD calculations existing at the time of the calculation are consistent with the $\chi \mathrm{PT}$ fit and its continuation to higher masses indicated by the dotted line.

have been successfully performed in the last year. Finally, partially-quenched calculations have been used to determine a charge-symmetry breaking quantity, the neutron-proton massdifference, from isospin-symmetric configurations [19].

Important highlights include:

\section{- Nucleon Axial Charge}

The nucleon axial charge, governing neutron $\beta$ decay, has been calculated for pion masses as light as $350 \mathrm{MeV}$ using domain wall valence quarks on a staggered fermion sea. Using chiral perturbation theory, analytic expressions for the mass and volume dependence were used to extrapolate to the physical pion mass and infinite volume, obtaining the axial charge to a precision of $6.8 \%$ and in agreement with experiment as shown in Fig 1.[12]

\section{- Isovector nucleon form factors}

Full QCD calculations with domain wall valence quarks on a staggered sea down to the chiral regime[20] dramatically display the the growth of the nucleon pion cloud as the pion mass decreases into the chiral regime. As seen in Fig. 2, the $\mathrm{F}_{1}$ form factor monotonically decreases toward the experimental result as the pion mass decreases and a chiral perturbation theory fit to the slope extrapolates to the experimental value. Similarly, as the pion mass decreases, these calculations also approach the experimental result for the ratio $F_{1} / F_{2}$ measured by polarization transfer experiments at JLab.

- Quark helicity and orbital angular momentum contribution to the nucleon spin

Recent lattice calculations provide the first glimpse of the origin of the nucleon spin in the chiral regime. The contribution of the spin of the up and down quarks to the total 

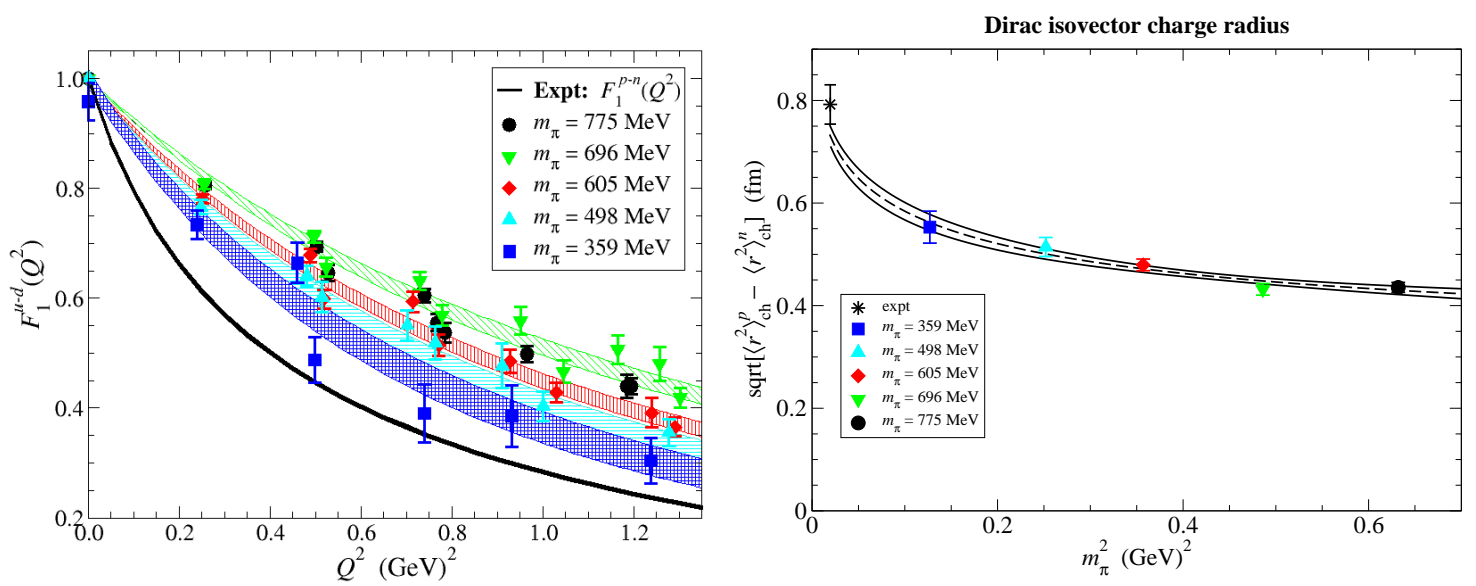

Figure 2: Isovector $\mathrm{F}_{1}$ form factor at five masses compared with experiment (left panel) and chiral extrapolation of form factor slope (right panel).

spin of the nucleon is given by the zeroth moment of the spin dependent structure function $\frac{1}{2} \Delta \Sigma=\frac{1}{2}\langle 1\rangle_{\Delta u+\Delta d}$ and by the Ji sum rule [21], the contribution of the quark orbital angular momentum is given by $L_{q}=\frac{1}{2}\left(A_{20}^{u+d}(0)+B_{20}^{u+d}(0)\right)-\frac{1}{2} \Delta \Sigma$. The dominant connected diagram contributions to these matrix elements has been calculated down to the chiral regime using domain wall valence quarks on a staggered sea[20] with the result that the fraction of the nucleon spin arising from quark helicity decreases from around $70 \%$ at $m_{\pi}=900 \mathrm{MeV}$ to approximately $50 \%$ at $350 \mathrm{MeV}$, and a negligible fraction comes from the total quark orbital angular momentum.

- Application of correlation-matrix techniques to lattice QCD: pure-gauge glueball and quenched nucleon spectrum.

Correlation matrix techniques offer a powerful means for determining the excitations of a theory. A dramatic and highly cited demonstration of the method was the determination, within the pure-gauge Yang-Mills theory, of the spectrum of glueballs, pure gluonic states characteristic of the non-Abelian nature of the theory[22, 23]. More recently, a basis of operators for baryon states has been developed [6, 7], so that an analogous program for states containing quarks is now within reach. Preliminary results are shown in Fig. 3.

\section{- Nature of Roper resonance.}

The Roper resonance, the lowest-lying radial excitation of the nucleon with a mass below that of the lightest negative-parity state, the $S_{11}(1535)$, is difficult to reconcile with phenomenological models of QCD. Employing the overlap-fermion formulation to reach unprecedentedly low pion masses, and using Bayesian analysis techniques, the mass of the Roper in the quenched approximation to QCD has been shown to approach that of the $S_{11}$ as the pion mass decreases[25].

- Radiative transition form factors and two-photon decays in charmonium. 

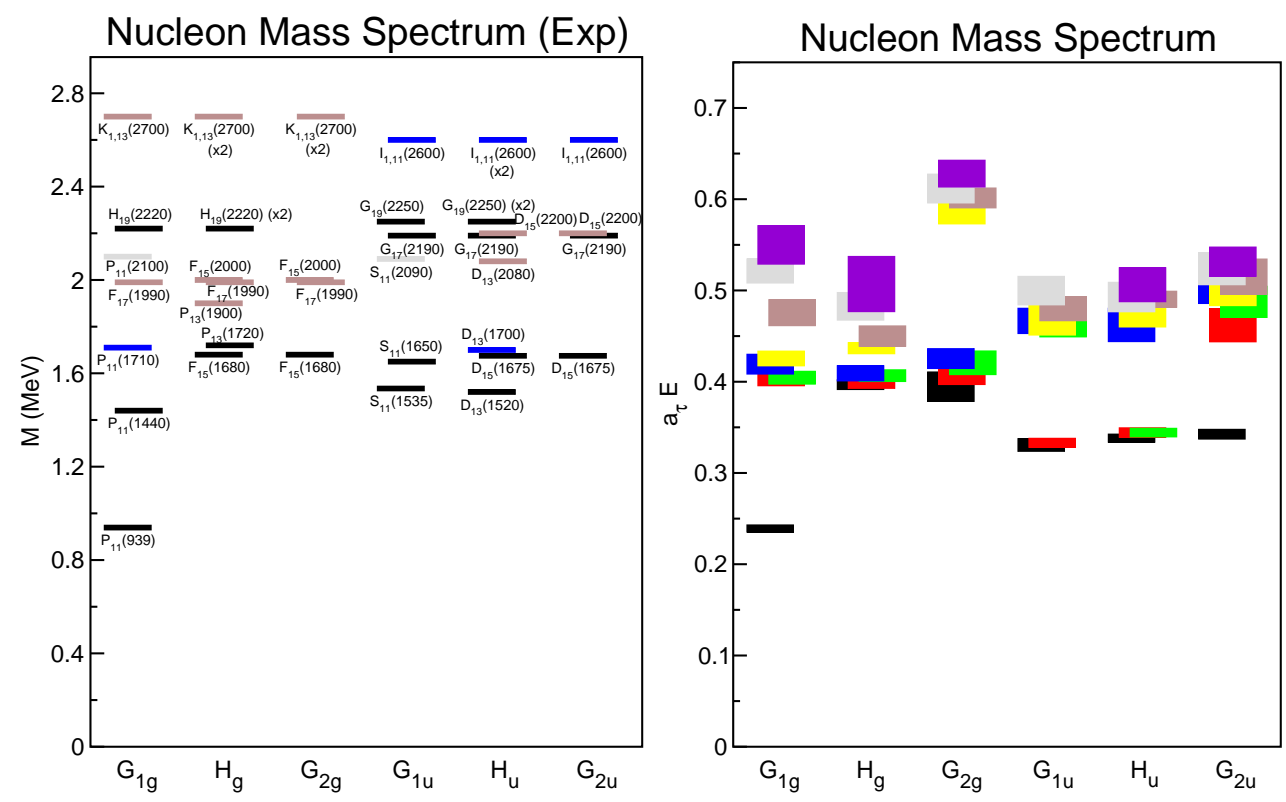

Figure 3: The left-hand panel shows the currently known spectrum from experiment, plotted according to the cubic symmetries allowed by the lattice; black denotes four-star states, blue denotes three-star states, tan denotes two-star states, and gray denotes a one-star state. The right-hand panel shows the nucleon spectrum in quenched QCD with $m_{\pi} \sim 700 \mathrm{MeV}$ from Ref. [24], where even at this unphysically large pion mass, one observes the emergence of patterns seen in the experimental spectrum.

The first calculation of the transition form factors between the lowest-lying charmonium states was performed, showing good agreement both with QCD-inspired models, and with experimental measurements[26]. This work lays the ground work for future computations of photocouplings both to hybrid states, and in the light-quark sector[26]. In a novel extension, the two-photon decay rate was computed[27].

\section{- Meson-Meson Scattering from Lattice QCD}

Meson-meson scattering lengths were calculated by the NPLQCD collaboration with domain-wall fermions on $N_{f}=2+1$ dynamical MILC sea configurations with pion masses down to $m_{\pi} \sim 290 \mathrm{MeV}$ [11], obtaining the results shown in Figure 4 . The first prediction of the $K \pi$ scattering lengths in both isospin channels was made possible by combining the lattice QCD calculation in the $I=3 / 2$ channel with chiral perturbation theory, the result of which is shown in Figure 4. To circumvent the Maiani-Testa theorem [28], which states that one cannot compute Green functions at infinite volume on a Euclidean lattice and recover S-matrix elements except at kinematic thresholds, this work computes the energy-eigenstates of the two particle system at finite volume to extract the scattering amplitude [29] and supersedes earlier CP-PACS calculations of $I=2 \pi \pi$ scattering with pion masses down to $m_{\pi} \sim 500 \mathrm{MeV}$.

\section{- NN Scattering from Lattice QCD}

The first studies of nucleon-nucleon scattering with fully-dynamical lattice QCD were 

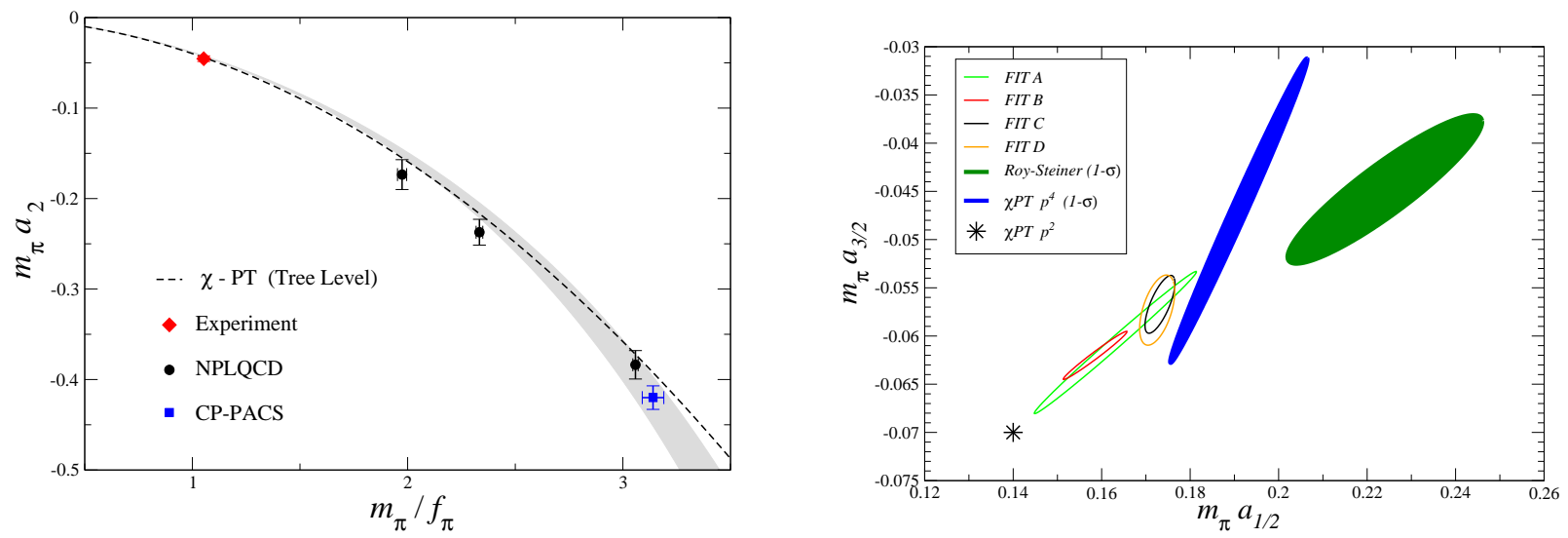

Figure 4: Meson-meson scattering lengths. The left panel shown $I=2 \pi \pi$ scattering lengths as a function of $m_{\pi} / f_{\pi}$ [11], while the right panel shows the prediction for the $K \pi$ scattering lengths at the physical pion mass [16].
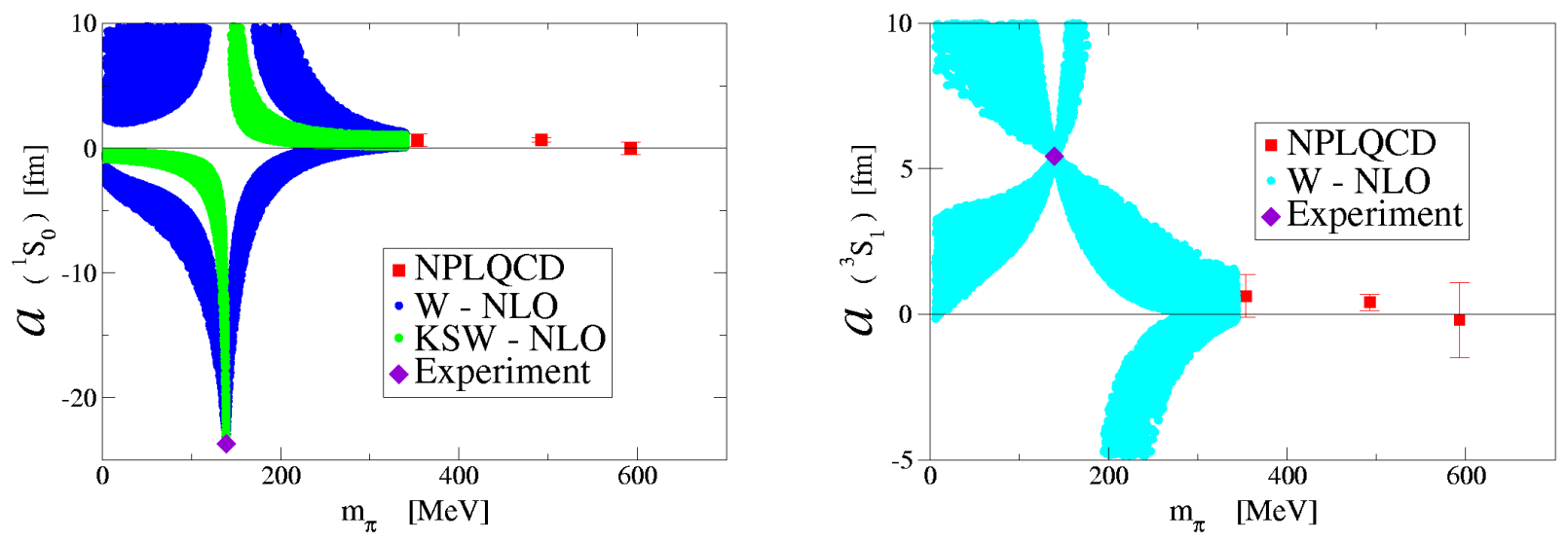

Figure 5: Results of the fully-dynamical lattice QCD calculations [17] (data points) and effective field theory chiral extrapolations (shaded regions).

performed this year.[17]. Although the pion masses used in the calculation $m_{\pi} \gtrsim 350 \mathrm{MeV}$ were too large to uniquely match to low-energy effective theory, including the physical scattering lengths led to the chiral behavior indicated in Figure 5, and demonstrating the power of the method for future calculations. In addition, the first calculations of hyperon-nucleon interactions were performed [18], in which is was shown that the scattering phase-shifts for elastic processes such as $n \Sigma^{-}$, of importance for the nuclear equation of state at high densities, can be extracted. 


\section{Future Opportunities}

The recent success in solving full QCD with dynamical quarks in the chiral regime opens an exciting range of research opportunities with the potential for significant impact on experiment, on our understanding of how QCD works, and allowing us to make reliable theoretical predictions where experiments are not possible and where other theoretical techniques fail. This section summarizes the prospects for high precision calculations of presently calculable observables, new observables and theoretical issues to be addressed, and anticipated milestones accessible with successive levels of computational resources.

\section{Precision computation of presently calculable observables}

Using the methodology and algorithms already developed and tested in calculations entering the chiral regime, the new computational resources becoming available in the coming five years will enable precision calculation of a broad range of important observables, ultimately reducing the combination of systematic and statistical errors to the level of a few percent. Key developments will include increasingly sophisticated use of partially quenched mixed-action chiral perturbation theory, the use of smaller lattice spacings to approach the continuum limit, larger volumes to approach the infinite-volume limit, smaller pion masses to approach the chiral limit, and nonperturbative operator renormalization.

In hadron structure, these high precision calculations will apply to a wide range of isovector operators that can be evaluated with connected diagrams, including electromagnetic form factors, moments of structure functions, generalized form factors corresponding to moments of generalized parton distributions, transition form factors and nucleon polarizabilities.

In hadron spectroscopy, the new computational resources will revolutionize our ability to compute the low-lying hadron spectrum with sufficient reliability to both guide and interpret the future experimental program. The use of anisotropic lattices, with temporal lattice spacing smaller than that in the spatial directions, enables the energies to be more precisely resolved, and is crucial for studies of the excitation spectrum. Capitalizing on the achievement of the HP2009 Milestone will require precise computations of the baryon resonance spectrum in lattice QCD. We have demonstrated our ability to extract several energies in preliminary studies of the spectrum. Delineating resonances and their quantum numbers from these energies will require that the computations be performed at several volumes and lattice spacings, and at sufficiently small values for the quark masses so that reliable chiral extrapolations can be made.

In the study of hadron interactions, the resources will enable precise calculations of many of the two-body processes of interest to nuclear and particle physicists at pion masses close to those of nature and at small lattice-spacings so that chiral and lattice-spacing extrapolations are reliable and independent of the quark discretization employed. These calculations can be performed with the lattices employed for the study of hadron structure and the hadron spectrum. It is likely that peta-scale resources will be required in order to make significant progress in computing nucleon-nucleon scattering at or very near the physical pion mass. 


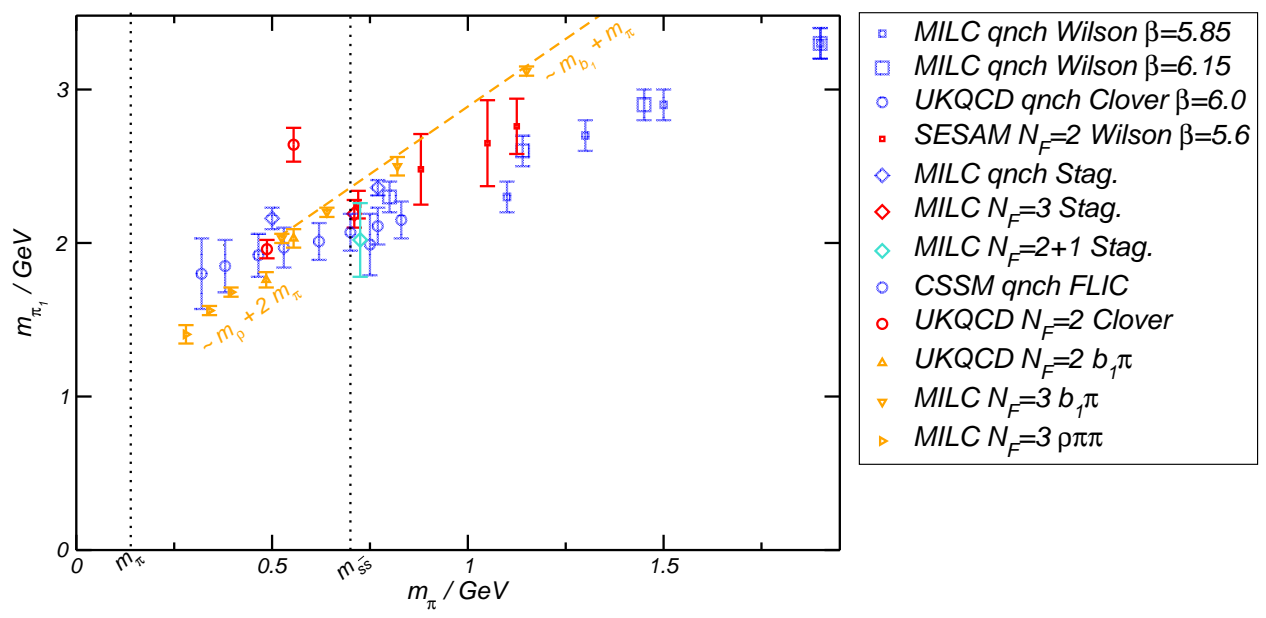

Figure 6: The figure shows a compendium of lattice studies of light $1^{-+}$hybrid mesons; the vertical lines correspond to the physical pion and $s \bar{s}$ pion masses, respectively. Also shown is the threshold corresponding to $b_{1} \pi$ production.

\section{New observables and theoretical issues}

Much of the intellectual vitality and excitement of the field centers on new theoretical developments and ideas for calculating important physical quantities that are presently inaccessible. Striking developments in recent years, such as the discovery of how to implement chiral symmetry on the lattice, highlight the impact of innovation in lattice field theory. Some of the key problems under intense investigation in hadronic physics are described below.

To calculate flavor-singlet matrix elements, in addition to the connected contributions, it is necessary to calculate disconnected contributions, which are typically several orders of magnitude more computationally expensive. Current development of eigenmode expansions and stochastic source techniques offer the prospect of calculating flavor singlet form factors, moments of quark distributions, and generalized form factors, thereby addressing the full range of experimental nucleon observables including strangeness contributions. Additional new calculations include gluon distributions, mixing of gluon and flavor singlet operators, operator mixing of higher moments of structure functions and generalized form factors, higher twist operators, the neutron electric dipole moment, and differences between moments of structure functions of a free neutron plus a free proton and a deuteron. Furthermore, these techniques will allow for the calculation of $I=0 \pi \pi$ scattering and $I=1 / 2 K \pi$ scattering.

The so-called "hybrid" mesons are emblematic of the mechanism of confinement. There have been only tentative explorations of the spectrum of light hybrid mesons, predominantly in the quenched approximation to QCD, as illustrated in Figure 6. We will be able to extend these computations from the relatively heavy quark masses used thus far to quark masses close to the physical light-quark masses. In this region, these heavier states can become resonances, lying above two-particle decay channels into which they can, in the continuum, decay, as illustrated in the figure. The first steps at calculating the decay width of the $\rho$ meson have recently been undertaken[30]; future resources will enable the extraction of decay-width 
information for exotic mesons.

Beyond establishing the spectrum, lattice QCD can compute their properties, expressed through the form factors and transition form factors. In the baryon sector, the first experimental transition form factors to resonance excitations are now appearing, and commensurate lattice computations will enable a picture of these resonances to emerge. The GlueX program in Hall D at Jefferson Laboratory aims to photoproduce hybrid and conventional meson resonances through the $t$-channel exchange of a conventional meson. Thus a knowledge of the photocouplings between conventional and hybrid mesons is crucial to estimating expected photoproduction rates. Such photocouplings, though estimated in QCD-inspired models, are unknown, and present a clear target for lattice QCD computations to make predictions vital for the experimental program.

The hyperon-nucleon interaction is terra incognita, but with important astrophysical implications, in particular, impacting the late time evolution of a supernova. Thus experimental programs in hypernuclear physics are gaining increasing importance. Furthermore, the YN interaction probes an additional aspect of the nuclear force, notably the role of valence strange quarks in the force between hadrons. Thus its study represents an exciting new opportunity for lattice QCD to work in concert with a vigorous hypernuclear physics experimental program. Finally, with the development of better algorithms, the potential between infinitely massive hadrons, such as B-mesons and $\Lambda_{b}$ baryons will become computable. These potentials will impact our understanding of the nucleon-nucleon potential.

\section{Milestones}

Based on present experience and algorithms, it is possible to extrapolate and estimate many of the lattice calculations that become feasible at successive levels of computational resources. All estimates below are expressed in terms of sustained Teraflops-years (the number of operations performed in one year of running at one sustained Teraflops). Motivated by DOE plans for leadership class machines with peak speed 100 Teraflops in 2007 and 1000 Teraflops (1 Petaflops) in 2008, and discussion of a follow-on machine sustaining 1 Petaflops, we have grouped milestones in categories of 10, 100, and 1000 Teraflops-years.

\section{Teraflops-years}

This level of resources will enable the completion of calculation of hadron structure observables with domain wall valence quarks on an improved staggered sea. Calculations of form factors, moments of quark distributions, generalized form factors and related operators at pion masses down to $250 \mathrm{MeV}$ at three lattice spacings, $\mathrm{a}=0.125,0.09$, and $0.06 \mathrm{fm}$, combined with mixed action chiral perturbation theory will enable controlled extrapolation in lattice spacing, lattice volume, and pion mass with high statistics. Given the past success in calculating $g_{A}$ to $6.8 \%$ accuracy, it is reasonable to expect a range of observables to be calculable at the level of $5-10 \%$. The smallest lattice spacing also has the virtue of enabling calculation of form factors up to the order of $10 \mathrm{GeV}^{2}$. This level of resources will also enable the beginning of fully chiral calculations using domain wall valence quarks on a domain wall sea, with $a=0.094 \mathrm{fm}$ 
and $m_{\pi}$ of the order of $300 \mathrm{MeV}$. Significant calculations of disconnected diagrams and gluon observables are also expected in this regime.

Our understanding of the hadron spectrum and hadronic interactions will be facilitated though calculations performed on anisotropic lattices, with volumes up to $4 \mathrm{fm}$, lattice spacings of $a=0.125$ and $0.10 \mathrm{fm}$, and pion masses down to $200 \mathrm{MeV}$. This will yield the first calculations in the light-quark regime of the spectrum of exotic meson masses, and the first predictions of the $\pi_{1}$ hybrid photocouplings to conventional mesons, providing vital input into the GlueX experimental program. The computation of the low-lying baryon resonance spectrum, including their decay widths, will confront the experimental analysis to yield insights into the degrees of freedom of QCD.

High precision calculations of $\pi \pi, K \pi$ and $K K$ scattering amplitudes will be possible, and predictions of the scattering lengths can be compared with upcoming experimental determinations. Investigations of $Y N$ scattering lengths will provide insight into the hyperon-nucleon interactions, about which there is a paucity of phenomenological knowledge, and complementing the experimental initiatives in hypernuclear physics.

\section{Teraflops-years}

At this level of resources, fully chiral structure calculations with both domain wall valence and sea quarks are possible for the same observables as above with $a=0.094 \mathrm{fm}, m_{\pi}$ of the order of $200 \mathrm{MeV}$, and significant statistics. For physical quantities such as the neutron electric dipole moment, where topology plays a fundamental role in connection with the $\Theta$ angle, an accurate, fully chirally symmetric calculation will be a significant breakthrough. Higher statistics on computationally demanding disconnected diagram and gluon observables are expected to have significant physics impact.

Calculations of the spectrum using anisotropic clover lattices will be possible at three lattice spacings of $a=0.08,0.10$ and $0.125 \mathrm{fm}$, and at pion masses as low as $180 \mathrm{MeV}$. Thus the spectrum both of baryons and mesons in the continuum limit will be known, and the transition form factors for the many of these states will become accessible.

The nucleon-nucleon scattering lengths will be known with sufficient precision at pion masses within the range of convergence of effective field theory so that an extrapolation to the physical pion mass will be possible. Furthermore, calculations of hyperon-nucleon scattering at a similar level of precision will be performed, and ambiguities in the nuclear equation of state, currently due to the lack of experimental data, will be greatly reduced. The first studies of the three-nucleon interaction will be performed, with an eye toward computing the nuclear reactions between the lightest elements. Exploratory studies of the direct computation of electroweak transition matrix elements in the two-nucleon sector will become possible.

\section{Petaflops-year}

At this scale of resources, we expect to reach the unprecedented milestone of carrying out calculations with chiral fermions at the physical pion mass. These calculations, combined 
with chiral perturbation theory analysis including higher masses, should enable precision calculations of many observables at the level of a few percent. Validated precision calculations of moments of generalized parton distributions can be used to complement experimental measurements of convolutions of generalized parton distributions and determine details of generalized parton distributions inaccessible to experiment alone. One can expect a detailed knowledge of the origin of the nucleon spin.

A precise computation of the deuteron binding energy and other properties of the deuteron at the physical pion mass may well be possible with 1 Petaflops-year, a remarkable achievement critical for satisfying the Department of Energy's milestone for 2014. The computation of nuclear reactions among the lightest nuclei may become possible with this magnitude of computing power.

\section{Conclusions}

Lattice QCD has a vital role in addressing questions that go to the heart of hadronic and nuclear physics, and to our understanding of the strong interaction. The last five years have witnessed the opening of new avenues for lattice QCD, most notably in the application to the nuclear force, and the emergence of new theoretical and algorithmic improvements that are vastly increasing the utility of lattice QCD in more established areas. The next five years afford the opportunity to exploit these advances to address many of the key questions in hadronic and nuclear physics, but this will only be accomplished with the appropriate investment both in computational resources, and the theoretical effort to exploit these resources.

\section{Acknowledgements}

This work was supported in part by DOE Office of Nuclear Physics contract DE-AC0506OR23177, under which Jefferson Science Associates, LLC, operates Jefferson Laboratory; JN and MJS are supported in part by DOE Office of Nuclear Physics grants DF-FC0294ER40818 and DE-FG03-97ER4014, respectively.

\section{References}

[1] D. Dolgov et al. [LHPC collaboration], full [arXiv:hep-lat/0201021].

[2] The LHC Collaboration: P. Hagler, et al., Phys. Rev D68, 034505 (2003).

[3] The LHC Collaboration: P. Hagler, et al., Phys. Rev. Lett. 93, 112001 (2004).

[4] C. Alexandrou, P. de Forcrand, Th. Lippert, H. Neff, J. W. Negele, K. Schilling, W. Schroers and A. Tsapalis, Phys. Rev D69, 114506 (2004). 
[5] C. Alexandrou, P. de Forcrand, H. Neff, J. W. Negele, W. Schroers and A. Tsapalis, Phys. Rev. Lett. 94, 021601 (2005).

[6] The LHPC Collaboration: S. Basak, et al., Phys. Rev D72, 094506 (2005).

[7] The LHPC Collaboration: S. Basak, et al., Phys. Rev D72, 074501 (2005).

[8] N. Mathur et al., Phys. Rev. D 70, 074508 (2004) [arXiv:hep-ph/0406196].

[9] O. Jahn, J. W. Negele and D. Sigaev, Proceedings of Science (Lattice 2005) 069 (2005).

[10] S. R. Beane, P. F. Bedaque, A. Parreno and M. J. Savage, Phys. Lett. B 585, 106 (2004) [arXiv:hep-lat/0312004].

[11] S. R. Beane, P. F. Bedaque, K. Orginos and M. J. Savage [NPLQCD Collaboration], Phys. Rev. D 73, 054503 (2006) [arXiv:hep-lat/0506013].

[12] R. G. Edwards et al. [LHPC Collaboration], Phys. Rev. Lett. 96, 052001 (2006) [arXiv:hep-lat/0510062].

[13] D. B. Renner et al. [LHPC Collaboration], J. Phys. Conf. Ser. 46, 152 (2006) [arXiv:hep-lat/0607008].

[14] C. Alexandrou, T. Leontiou, J. W. Negele and A. Tsapalis, arXiv:hep-lat/0607030.

[15] The LHPC Collaboration: F.D.R. Bonnet, et al., Phys. Rev D72, 054506 (2005).

[16] S. R. Beane, P. F. Bedaque, T. C. Luu, K. Orginos, E. Pallante, A. Parreno and M. J. Savage, arXiv:hep-lat/0607036.

[17] S. R. Beane, P. F. Bedaque, K. Orginos and M. J. Savage, Phys. Rev. Lett. 97, 012001 (2006) [arXiv:hep-lat/0602010].

[18] S. R. Beane, P. F. Bedaque, T. C. Luu, K. Orginos, E. Pallante, A. Parreno and M. J. Savage, arXiv:hep-lat/0612026.

[19] S. R. Beane, K. Orginos and M. J. Savage, arXiv:hep-lat/0605014.

[20] R. G. Edwards et al., arXiv:hep-lat/0610007.

[21] X. D. Ji, Phys. Rev. Lett. 78, 610 (1997) [arXiv:hep-ph/9603249].

[22] C. J. Morningstar and M. J. Peardon, Phys. Rev. D 60, 034509 (1999) [arXiv:heplat/9901004].

[23] Y. Chen et al., Phys. Rev. D 73, 014516 (2006) [arXiv:hep-lat/0510074].

[24] A. Lichtl, arXiv:hep-lat/0609019.

[25] N. Mathur et al., Phys. Lett. B 65, 137 (2005) [arXiv:hep-ph/0306199]. 
[26] J. J. Dudek, R. G. Edwards and D. G. Richards, Phys. Rev. D 73, 074507 (2006) [arXiv:hep-ph/0601137].

[27] J. J. Dudek and R. G. Edwards, Phys. Rev. Lett. 97, 172001 (2006) [arXiv:hep$\mathrm{ph} / 0607140]$.

[28] L. Maiani and M. Testa, Phys. Lett. B 245, 585 (1990).

[29] M. Luscher, Commun. Math. Phys. 105, 153 (1986).

[30] S. Aoki et al. [CP-PACS Collaboration], arXiv:hep-lat/0610020. 